\title{
Strangeness and heavy flavours within the ALICE experiment
}

\author{
Iouri Belikov (for the ALICE Collaboration) \\ IPHC-DRS, UDS, CNRS, IN2P3, 67037 Strasbourg, France \\ E-mail: Iouri.Belikov@IReS.in2p3.fr
}

\begin{abstract}
The ALICE experiment at CERN is now ready to take data in protonproton and ultra-relativistic heavy-ion collisions at LHC. This review starts with a description of the ALICE experimental setup and its excellent tracking and particle identification capabilities. Then, we will discuss the ways to probe the hot and dense medium created at multi- $\mathrm{TeV}$ energies by measuring the yields and kinematic characteristics of strange and heavy-flavour particles produced in these collisions.
\end{abstract}




\section{Introduction}

A Large Ion Collider Experiment (ALICE) [1] at CERN is a general-purpose heavy-ion experiment designed to study the physics of strongly interacting matter in nucleusnucleus collisions at the LHC. The centre-of-mass energy per nucleon pair will be $\sqrt{s_{\mathrm{NN}}}=5.5 \mathrm{TeV}$ for the $\mathrm{Pb}-\mathrm{Pb}$ system, and the energy density reached will be of the order of a few tens $\mathrm{GeV} / \mathrm{fm}^{3}$. Under these conditions, a deconfined state of quarks and gluons, the quark-gluon plasma, is expected to be formed. In addition to heavy systems, the ALICE Collaboration will study collisions of lower-mass ions and protons (both pp and $\mathrm{pA}$ ), which will provide the reference data for the nucleus-nucleus collisions, and will also allow for a number of genuine pp and pA physics studies.

The detector consists of a central part (see Fig. 1), which measures hadrons, electrons and photons, and of a forward spectrometer to measure muons. The central part, which covers polar angles from $45^{\circ}$ to $135^{\circ}$ is embedded in the large L3 solenoidal magnet. It consists of: an Inner Tracking System (ITS) of high-resolution silicon detectors; a cylindrical Time-Projection Chamber (TPC); three particle identification arrays of: Time-Of-Flight (TOF) detector, Transition-Radiation Detector (TRD) and a single-arm ring imaging Cherenkov (HMPID); and two electromagnetic calorimeters (PHOS and EMCAL). The forward muon spectrometer (covering polar angles $\theta=2^{\circ}-$ $9^{\circ}$ ) consists of a complex arrangement of absorbers, a large dipole magnet, and fourteen planes of tracking and triggering chambers. Several smaller detectors for global event characterization and triggering are located at forward angles.

With such a design, ALICE is capable of correlating measurements done at central and forward rapidities.

\section{ALICE detector performance}

The apparatus was optimized for a charged-particle density $\mathrm{d} N_{\mathrm{ch}} / \mathrm{d} y=4000$ and its performance has been checked in detailed simulations up to $\mathrm{d} N_{\mathrm{ch}} / \mathrm{d} y=8000$. The track reconstruction efficiency in the acceptance of TPC is about $80 \%$ down to $p_{\mathrm{T}}=0.2 \mathrm{GeV} / c$, and it is limited by particle decays and absorption in the material. For the tracks with $p_{\mathrm{T}}$ above $1 \mathrm{GeV} / c$, the reconstruction efficiency reaches $90 \%$, which is constrained by the dead zones between the TPC sectors.

The typical momentum resolution obtained with the magnetic field of $0.5 \mathrm{~T}$ is $1 \%$ at $p_{\mathrm{T}}=1 \mathrm{GeV} / c$. At lower momenta, it is dominated by the energy-loss fluctuations and multiple scattering in the material. The material budget of ITS and TPC together is about $15 \%$ of $\mathrm{X}_{0}$. At high momenta, the momentum resolution is determined by the space-point precision and the quality of the calibration and alignment of the tracking detectors (ITS, TPC and TRD). At $p_{\mathrm{T}}=100 \mathrm{GeV} / c$, the target momentum resolution is $3-5 \%$ for the tracks reconstructed in all the tracking detectors.

An essential part of the ALICE physics program involves the measurements of charm and beauty hadrons. The detection of these short-living particles (as an example, 


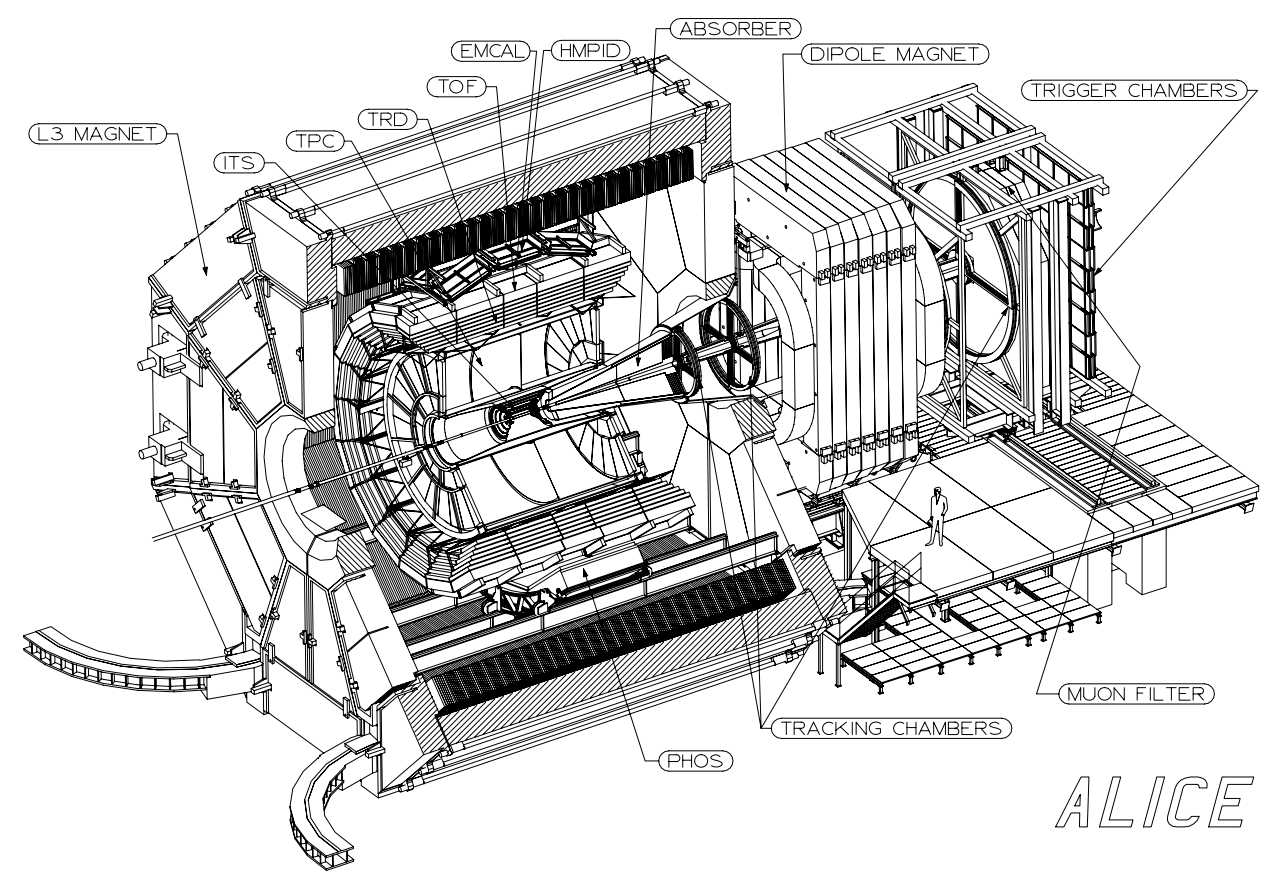

Figure 1. Schematic layout of the ALICE detector.

$c \tau$ for the $\mathrm{D}^{0}$ meson is $123 \mu \mathrm{m}$ ) relies on the resolution of the reconstructed track impact parameters with respect to the primary vertex, which has to be of the order of the particle's $c \tau$, or better. In ALICE, the impact parameter resolution is mainly defined by the two innermost pixel layers of ITS. For the events with a number of tracks bigger than 100, the resolution on the transverse impact parameter is below $60 \mu \mathrm{m}$ for the tracks with a momentum above $1 \mathrm{GeV} / c$. For the events with lower track multiplicities, the contribution from the precision of the primary vertex reconstruction becomes nonnegligible. But, even in this case, the transverse impact parameter resolution is always better than $100 \mu \mathrm{m}$ at $p_{\mathrm{T}}>1 \mathrm{GeV} / c$.

Using virtually all known experimental techniques, the ALICE detector has excellent particle identification capabilities. Combining the $\mathrm{d} E / \mathrm{d} x$ from ITS, TPC and TRD, the time-of-flight from TOF, and the Cherenkov angle from HMPID, ALICE can identify charged pions, kaons and protons in the momentum range from $0.2 \mathrm{GeV} / c$ to about $5 \mathrm{GeV} / c$ on a track-by-track basis. Statistically, the charged hadrons can be identified up to a few tens $\mathrm{GeV} / c$ using the relativistic rise of $\mathrm{d} E / \mathrm{d} x$ in TPC. Electrons above $1 \mathrm{GeV} / c$ are identified by transition radiation registered in TRD.

In 2008 and 2009, ALICE has collected a few million cosmic tracks suitable for the calibration and alignment of the detectors. The preliminary results are quite encouraging. The alignment procedures in ITS result in an average impact parameter resolution for cosmic tracks of the order of $50 \mu \mathrm{m}$, to be compared with $40 \mu \mathrm{m}$ observed in Monte Carlo events simulated with perfectly aligned detectors [2]. The $\mathrm{d} E / \mathrm{d} x$ resolution in TPC is $5.7 \%$, with the theoretically expected limit of $\sim 5.5 \%$. The achieved time resolution in TOF is about $110 \mathrm{ps,} \mathrm{which} \mathrm{is} \mathrm{within} \mathrm{the} \mathrm{range} \mathrm{of} 60-120$ ps resolution 
used in the Monte Carlo performance studies.

\section{Strangeness program}

\subsection{Topological reconstruction of the strange hadrons}

Strange particles in ALICE are reconstructed via their decay topologies: charged kaons as kinks, $\Lambda$ and $\mathrm{K}_{s}^{0}$ as V0s, and $\Xi$ and $\Omega$ as cascade decays. Depending on the actual slope of the $p_{\mathrm{T}}$ spectra and taking into account the reconstruction rates estimated with Monte Carlo, ALICE will have enough statistics of kaons and $\Lambda^{\prime}$ 's up to $p_{\mathrm{T}}=10-15 \mathrm{GeV} / c$, and $\Xi$ and $\Omega$ up to $p_{\mathrm{T}}=7-10 \mathrm{GeV} / c$ for both $10^{7}$ central $\mathrm{Pb}-\mathrm{Pb}$ and $10^{9}$ minimum bias pp collisions (one year of data taking under nominal conditions) [3].

\subsection{In-medium enhancement and the canonical suppression}

If a deconfined state of quarks and gluons is formed in heavy-ion collisions, strangeness production was predicted to be enhanced [4], and this was observed at SPS [5]. This can happen due to several reasons, like for a example lower kinematic threshold for the in-medium production of $s$-quarks and presence of additional production channels $g g \rightarrow s \bar{s}$, which are absent in the hadronic state. This excess of produced strangeness is preserved at the subsequent hadronization and results in a higher number of strange particles in the final state, than expected in a purely hadronic scenario.

The production of strangeness in heavy-ion collisions has to be compared with that in pp. However, the small systems require the strangeness to be conserved in a smaller correlation volume. In the language of statistical mechanics, the grand canonical ensemble limit (possibly reached in high-multiplicity heavy-ion reactions) is not applicable to the pp collisions. In this case, the canonical ensemble description becomes more appropriate. The lack of the available phase space leads to the canonical suppression of the strangeness production in small colliding systems [6].

The fact that this constraint is removed in heavy-ion collisions is not trivial. If these collisions were nothing else but a simple superposition of independent nucleon-nucleon interactions, the canonical suppression would still be in place, because the correlation volume would still be small. For this not to happen, there must exist a mechanism for long-range communication within the system, as it is expected if the partons become free in a quark-gluon plasma.

The statistical models based on canonical ensemble predict a fast saturation of the enhancement with the event centrality, which is not what was observed at RHIC. Also the magnitude of the enhancement decrease with increasing energy is weaker than it was predicted [7].

Measuring the strangeness enhancement as a function of centrality in nucleusnucleus collisions, and as the function of multiplicity in pp collisions at the LHC energies with ALICE will provide an important piece of information for constraining the models. The studies of the $\phi$ meson enhancement and its excitation function are of particular 
interest, because the production of this particle is not canonically suppressed (due to the $s \bar{s}$ structure of this meson).

\subsection{Collective effects at the quark level and mechanisms of strangeness production}

Topological reconstruction of the strange particles $\left(\mathrm{K}_{\mathrm{S}}^{0}\right.$ and $\Lambda$ ) gives us the unique possibility to distinguish between baryons and mesons at the momenta above the reach of the conventional particle identification methods. The $\Lambda / \mathrm{K}_{\mathrm{S}}^{0}$ ratio measured in $\mathrm{Au}-\mathrm{Au}$ collisions by STAR shows an excess of the baryon production with respect to the meson production within the range $1.5<p_{\mathrm{T}}<4.5 \mathrm{GeV} / c$ [8]. This excess increases with the collision centrality. A possible explanation of the phenomenon involves the collective effects at the quark level: the transverse radial flow and recombination.

The recombination models predict that the $\Omega$ production in heavy-ion collisions should be dominated by recombination of three thermal quarks. This should result in a thermal shape of the momentum spectrum and absence of correlations with jets. The first prediction was confirmed by the measurements, whereas the second was not. An interesting solution to this $\Omega$ puzzle was proposed in [9], suggesting that $\Omega$ 's are produced not in the jets themselves but rather in the ridge associated with them.

Due to its excellent capabilities for both strange particle and full jet reconstruction, ALICE will be able to check these ideas directly. High statistics of reconstructed strange particles will allow for studying the $\Lambda / K_{\mathrm{S}}^{0}$ ratio in pp collisions as a function of multiplicity, which, at the LHC energies, may also start revealing non-trivial collective effects at the partonic level.

\subsection{In-medium modifications of resonance properties}

Resonances with lifetimes comparable to that of the deconfined phase (such as $\mathrm{K}^{*}(890)^{0}$, $\phi(1020))$ may change their properties (mass, width) when they are produced in the dense medium. This may happen due to the final state interaction (which can be sorted out by comparing the results obtained in the hadronic and leptonic decay channels) or due to the partial chiral symmetry restoration.

The ALICE detector will be able to register these resonances with sufficient statistics and a mass resolution of the order of a few $\mathrm{MeV} / \mathrm{c}^{2}$ (see Fig. 2).

\section{Heavy-flavour program}

\subsection{Reconstruction of the open-heavy-flavour particles and quarkonia}

The D mesons in ALICE will be topologically reconstructed in the central detectors. The two most promising channels are $\mathrm{D}^{0} \rightarrow \mathrm{K}^{-} \pi^{+}$and $\mathrm{D}^{+} \rightarrow \mathrm{K}^{-} \pi^{+} \pi^{+}$. The detection strategy to cope with the large combinatorial background is based on the selection of displaced-vertex topologies and good colinearity between the reconstructed D meson momentum and the flight line [3]. An invariant-mass analysis is then used to extract 

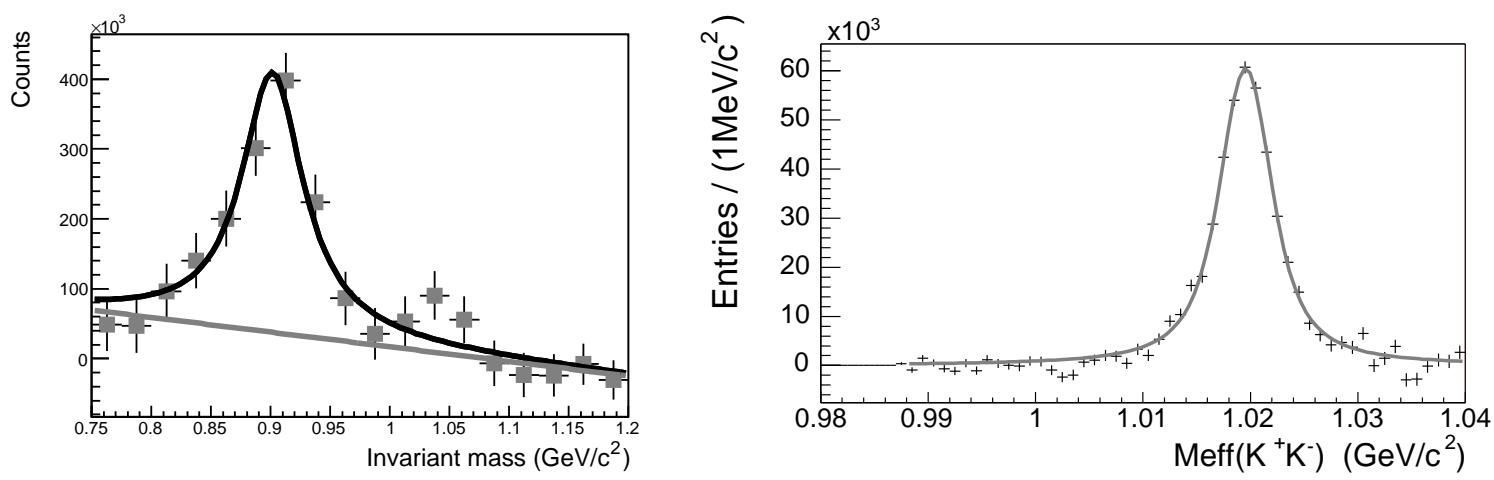

Figure 2. Background subtracted invariant-mass spectra of $\mathrm{K}^{*}(890)^{0}$ (left panel) and $\phi(1020)$ (right panel) reconstructed by ALICE in simulated central $\mathrm{Pb}-\mathrm{Pb}$ events [3].

the signal yield. For one year of data taking at nominal luminosity, the accessible $p_{\mathrm{T}}$ range for the $\mathrm{D}^{0}$ is $1-20 \mathrm{GeV} / c$ in $\mathrm{Pb}-\mathrm{Pb}$ and $0.5-20 \mathrm{GeV} / c$ in pp, with statistical errors better than $15-20 \%$ at high $p_{\mathrm{T}}$. Similar capability is expected for the $\mathrm{D}^{+}$, though at present the statistical errors are estimated only in the range $1<p_{\mathrm{T}}<8 \mathrm{GeV} / c$.

The production of open beauty can be studied in the central part of ALICE by detecting the semi-electronic decays of beauty hadrons, mostly B mesons. Given that electrons from beauty have an average impact parameter $d_{0} \sim 500 \mu \mathrm{m}$ and a hard $p_{\mathrm{T}}$ spectrum, it is possible to obtain a high-purity sample with a strategy that relies on the electron identification by combining $\mathrm{d} E / \mathrm{d} x$ (TPC) and transition radiation (TRD) information, and the impact parameter cut reducing the charm-decay component and rejecting misidentified $\pi^{ \pm}$and $e^{ \pm}$from Dalitz decays and $\gamma$ conversions. Beauty production can be measured also in the ALICE muon spectrometer, by analyzing the single-muon $p_{\mathrm{T}}$ distribution and the opposite-sign di-muons invariant mass distribution.

ALICE will be able to measure the charmonia and bottomonia production both at mid-rapidity (in di-electron channels) and at forward rapidities (in di-muon channels). In both channels the acceptance extends down to zero transverse momentum, and the mass resolution of $\sim 90 \mathrm{GeV}$ is sufficient to separate the $\Upsilon$ states (see Fig. 3 ).

\subsection{Mass and colour dependence of the in-medium energy losses}

When traversing the dense matter created in nucleus-nucleus collisions, the initiallyproduced hard partons loose energy. A substantial part of this energy loss is due to the medium-induced gluon radiation. Experiments at RHIC have shown that the nuclear modification factor of particle distributions, $R_{\mathrm{AA}}=\frac{1}{<N_{\mathrm{coll}}>} \frac{\mathrm{d} N_{\mathrm{AA}} / \mathrm{d} p_{\mathrm{T}}}{\mathrm{d} N_{\mathrm{pp}} / \mathrm{d} p_{\mathrm{T}}}$, is a sensitive observable for the study of the interaction of the hard partons with the medium produced in nucleus-nucleus collisions. Quarks are predicted to loose less energy than gluons (that have a higher colour charge) and, in addition, the "dead-cone effect" is expected to reduce the energy loss of massive quarks with respect to light partons [10, 11]. Therefore, one should observe a pattern of progressively decreasing $R_{\mathrm{AA}}$ suppression when going from the mostly gluon-originated light-flavour hadrons $\left(\mathrm{h}^{ \pm}\right.$or $\left.\pi^{0}\right)$ to $\mathrm{D}$ and to B mesons: 

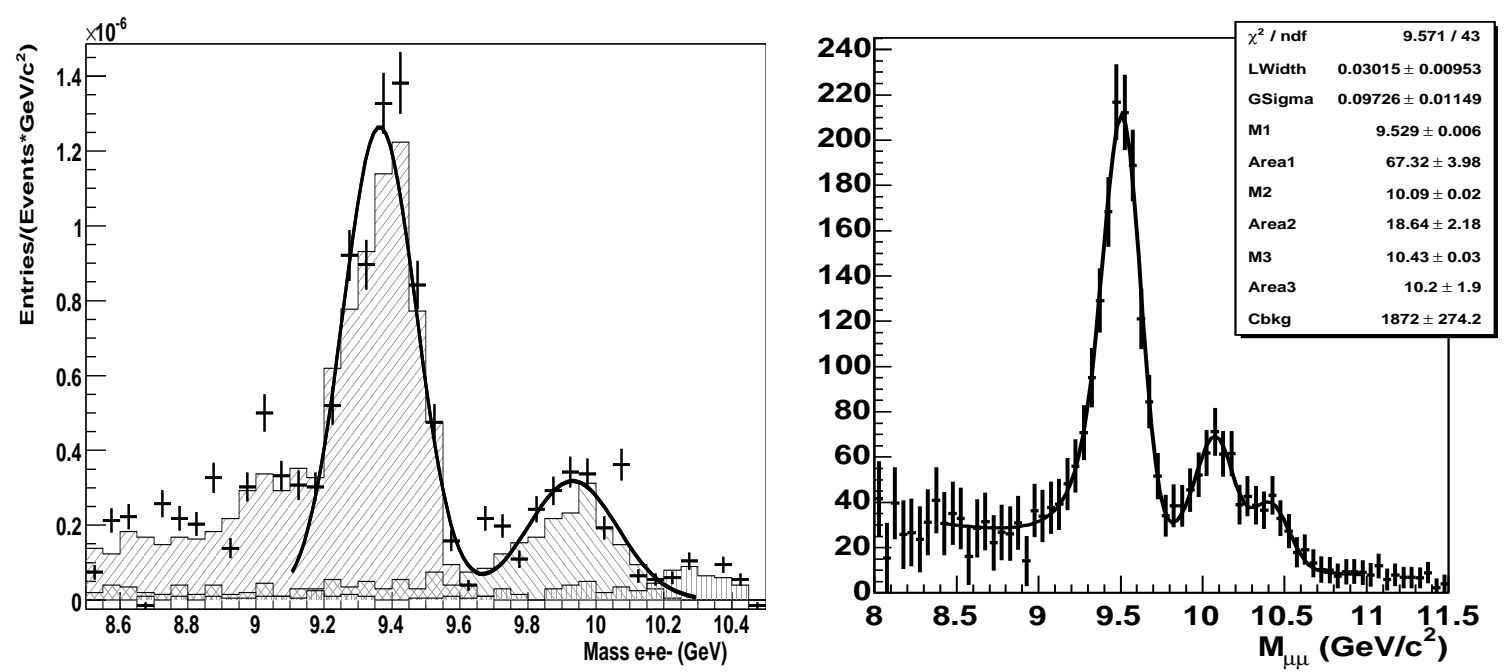

Figure 3. The signal of $\Upsilon$ states, reconstructed by ALICE [3] in the di-electron (left panel) and in the di-muon (right panel) channel, in one month of data-taking.

$R_{\mathrm{AA}}^{\mathrm{h}} \leq R_{\mathrm{AA}}^{\mathrm{D}} \leq R_{\mathrm{AA}}^{\mathrm{B}}$. The possible enhancement, with respect to unity, of ratios between these $R_{\mathrm{AA}}$ 's has been suggested [12] as a sensitive observable to test the colour-charge $\left(R_{\mathrm{D} / \mathrm{h}}=R_{\mathrm{AA}}^{\mathrm{D}} / R_{\mathrm{AA}}^{\mathrm{h}}\right)$ and mass $\left(R_{\mathrm{B} / \mathrm{D}}=R_{\mathrm{AA}}^{\mathrm{B}} / R_{\mathrm{AA}}^{\mathrm{D}}\right)$ dependence of parton energy loss.

At LHC, due to high production rates expected for heavy flavour particles and also jets, the measurements of the in-medium energy losses can be performed in a new and promising way. With its high potential for detecting the charm and beauty combined with the capabilities for full jet reconstruction, ALICE will address these effects also by studying directly the properties of heavy-flavour-tagged jets.

In addition to the partonic energy loss studies, the precise detection of the opencharm (open-beauty) hadrons is of an essential interest as a natural reference for the quarkonia production measurement.

\subsection{In medium suppression and regeneration of the quakonium states}

In nucleus-nucleus collisions, quarkonium suppression is expected to occur due to the Debye screening of colour charge in the deconfined matter. Such a suppression was already observed at SPS [13]. However, at higher energies (RHIC, LHC) the situation becomes more complicated, because the charmonia can be regenerated in the hot medium by recombination $[14,15]$. On the other hand, at LHC energies, the statistics of registered $\Upsilon$ 's is for the first time expected to be sufficient for detailed suppression studies. In this case, due to the much higher mass, the influence of the regeneration should be negligible.

Thus, the most interesting aspect of the quarkonia studies with ALICE will be understanding the interplay between colour-screening-induced suppression and regeneration for $\mathrm{J} / \psi$ production in a medium containing on the order of $100 c \bar{c}$ pairs, 
and measuring the medium effects on the bottomonia resonances.

\section{Conclusions}

We have discussed how the measurements of yields and kinematic properties of strange and heavy-flavour particles, abundantly produced at LHC energies, will allow to address several issues of the physics of deconfined matter. They provide tools for testing the thermodynamical equilibrium (by measuring the particle ratios) and collective effects at the parton level (by studying the mechanisms of particle production), tools for observing the possible chiral symmetry restoration (via detection of the in-medium changes of the resonance properties). By measuring the parton energy loss and its predicted mass dependence we will probe the properties of the QCD medium produced in the collisions, and the observations of the sequential dissociation patterns of quarkonia states will provide information about the temperature of the system.

Owing to its excellent tracking, vertexing and particle identification performance, ALICE is well equipped for strangeness and heavy-flavour physics. The low overall material budget of the central detectors makes possible charm and beauty detection down to $p_{\mathrm{T}} \sim 0$. The experiment has the capabilities to study correlations between strange, charm and beauty particles and jets. The measurements will be done at central and forward rapidities simultaneously.

All these advantages, in combination with high statistics of strange and heavyflavour particles and well developed jets expected at LHC, will allow us to explore in detail this rich field of high-energy physics.

\section{References}

[1] F. Carminati et al. [ALICE Collaboration], J. Phys. G 30, 1517 (2004).

[2] C. Bombonati et al., ALICE Internal Note ALICE-INT-2009-035.

[3] B. Alessandro et al. [ALICE Collaboration], J. Phys. G 32, 1295 (2006).

[4] J. Rafelski and B. Muller, Phys. Rev. Lett. 48, 1066 (1982).

[5] E. Andersen et al. [WA97 Collaboration], Phys. Lett. B 449 (1999) 401.

[6] S. Hamieh, K. Redlich and A. Tounsi, Phys. Lett. B 486, 61 (2000) [arXiv:hep-ph/0006024].

[7] B. I. Abelev et al. [STAR Collaboration], Phys. Rev. C 77, 044908 (2008) [arXiv:0705.2511 [nuclex]].

[8] M. A. C. Lamont, Eur. Phys. J. C 49 (2007) 35.

[9] R. C. Hwa, J. Phys. G 34 (2007) S789 [arXiv:nucl-th/0701018].

[10] Yu.L. Dokshitzer and D.E. Kharzeev, Phys. Lett. B 519, 199 (2001) [arXiv:hep-ph/0106202].

[11] N. Armesto, C.A. Salgado and U.A. Wiedemann, Phys. Rev. D 69, 114003 (2004) [arXiv:hep$\mathrm{ph} / 0312106]$.

[12] N. Armesto, A. Dainese, C. A. Salgado and U. A. Wiedemann, Phys. Rev. D 71 (2005) 054027 [arXiv:hep-ph/0501225].

[13] B. Alessandro et al. [NA50 Collaboration], Eur. Phys. J. C 39, 335 (2005) [arXiv:hep-ex/0412036].

[14] A. Andronic, P. Braun-Munzinger, K. Redlich and J. Stachel, Phys. Lett. B 652, 259 (2007) [arXiv:nucl-th/0701079].

[15] A. Andronic, P. Braun-Munzinger, K. Redlich and J. Stachel, Nucl. Phys. A 789, 334 (2007) [arXiv:nucl-th/0611023]. 